\title{
RESEARCH
}

Open Access

\section{Microbiomes attached to fresh perennial ryegrass are temporally resilient and adapt to changing ecological niches}

Sharon A. Huws ${ }^{1,2^{*}}$ D, Joan E. Edwards ${ }^{2,3,4}$, Wanchang Lin², Francesco Rubino ${ }^{1}$, Mark Alston ${ }^{5}$, David Swarbreck ${ }^{5}$, Shabhonam Caim ${ }^{6}$, Pauline Rees Stevens' ${ }^{2}$, Justin Pachebat ${ }^{2}$, Mi-Young Won'1, Linda B. Oyama ${ }^{1,2}$, Christopher J. Creevey ${ }^{1,2+}$ and Alison H. Kingston-Smith ${ }^{2+}$

\begin{abstract}
Background: Gut microbiomes, such as the rumen, greatly influence host nutrition due to their feed energyharvesting capacity. We investigated temporal ecological interactions facilitating energy harvesting at the fresh perennial ryegrass (PRG)-biofilm interface in the rumen using an in sacco approach and prokaryotic metatranscriptomic profiling.

Results: Network analysis identified two distinct sub-microbiomes primarily representing primary $(\leq 4 \mathrm{~h})$ and secondary ( $\geq 4 \mathrm{~h}$ ) colonisation phases and the most transcriptionally active bacterial families (i.e Fibrobacteriaceae, Selemondaceae and Methanobacteriaceae) did not interact with either sub-microbiome, indicating non-cooperative behaviour. Conversely, Prevotellaceae had most transcriptional activity within the primary sub-microbiome (focussed on protein metabolism) and Lachnospiraceae within the secondary sub-microbiome (focussed on carbohydrate degradation). Putative keystone taxa, with low transcriptional activity, were identified within both sub-microbiomes, highlighting the important synergistic role of minor bacterial families; however, we hypothesise that they may be 'cheating' in order to capitalise on the energy-harvesting capacity of other microbes. In terms of chemical cues underlying transition from primary to secondary colonisation phases, we suggest that Al-2-based quorum sensing plays a role, based on LuxS gene expression data, coupled with changes in PRG chemistry.

Conclusions: In summary, we show that fresh PRG-attached prokaryotes are resilient and adapt quickly to changing niches. This study provides the first major insight into the complex temporal ecological interactions occurring at the plant-biofilm interface within the rumen. The study also provides valuable insights into potential plant breeding strategies for development of the utopian plant, allowing optimal sustainable production of ruminants.
\end{abstract}

Keywords: Rumen, Bacteria, Archaea, Biofilm, Microbiome, Temporal, Colonisation, Metatranscriptome, Ecology, Niche

\footnotetext{
*Correspondence: S.huws@qub.ac.uk

${ }^{\dagger}$ Christopher J. Creevey and Alison H. Kingston-Smith contributed equally to this work.

'Institute of Global Food Security, School of Biological Sciences, Queen's University Belfast, 19 Chlorine Gardens, Belfast BT9 5DL, Northern Ireland, UK ${ }^{2}$ Institute of Biological, Environmental and Rural Sciences, Aberystwyth University, Aberystwyth SY23 3FG, UK

Full list of author information is available at the end of the article
} 4.0 International License, which permits use, sharing, adaptation, distribution and reproduction in any medium or format, as long as you give appropriate credit to the original author(s) and the source, provide a link to the Creative Commons licence, and indicate if changes were made. The images or other third party material in this article are included in the article's Creative Commons licence, unless indicated otherwise in a credit line to the material. If material is not included in the article's Creative Commons licence and your intended use is not permitted by statutory regulation or exceeds the permitted use, you will need to obtain permission directly from the copyright holder. To view a copy of this licence, visit http://creativecommons.org/ licenses/by/4.0/. The Creative Commons Public Domain Dedication waiver (http://creativecommons.org/publicdomain/zero/1. 0/) applies to the data made available in this article, unless otherwise stated in a credit line to the data. 


\section{Introduction}

Vertebrates play host to a complex gut microbiome, generally dominated by a few well-studied groups, but with a large ensemble of minor microbial species whose contribution are only recently starting to be revealed [1-4]. Whilst it is generally accepted that distinct microbiomes exist in distal locations in the gut, it is not clear whether a single location could simultaneously play host to multiple sub-microbiomes with distinct temporal and/or functional roles. Neither do we fully understand the importance of ecological interactions in these environments. Elucidating these temporal niche-specialised interactions could drive the generation of new strategies for targeted manipulation of vertebrate gut microbiomes for therapeutic, productivity or environmental benefits.

The rumen is a case in point and is the largest compartment of the ruminant forestomach, housing a complex microbiome that has a major impact on host nutrition and health [5]. This anoxic microbial ecosystem has evolved to harvest energy from largely recalcitrant, complex plant carbohydrates [5-9]. Rumen microbes commonly exist as biofilms on feed particles consumed by the host [10-13] and microbes within biofilms have been shown to interact intimately and influence each other's evolutionary fitness in many ecological niches [9, 14-16]. Indeed, whilst the rumen microbes generally function symbiotically, they also compete against each other for evolutionary advantage [9, 17].

Using rrn operon-based approaches and microscopy, we have previously demonstrated that rumen bacteria and anaerobic fungi attach rapidly to PRG $[6,18,19]$, with biofilms being evident within 5 min [11]. Colonisation by bacteria thereafter is biphasic, with primary $(\leq 4$ h) and secondary ( $\geq 4 \mathrm{~h}$ ) colonisation phases previously described based on metataxonomy $[10,20]$. We hypothesise that these distinct temporal bacterial colonisation phases represent the interaction of multiple, coherent and yet temporally distinct sub-microbiomes. Whilst these interactions are frequently categorised as positive (e.g. mutualism), neutral (i.e. resulting in no effect) or negative (e.g. competition) [21, 22], it is becoming clear that ecological interactions in highly complex environments can also result in combinations of positive, neutral and negative outcomes, for example amensalism in which the actor experiences no benefit or detriment and the recipient experiences a negative outcome or commensalism when the converse is true [21]. Added to this, keystone taxa are likely to have an important role mediating mutually beneficial interactions which may be disproportionate to their population size and the loss of these taxa would have a profound impact upon the ecosystem [1]. Finally, cheating behaviour is also likely to exist in such complex microbiomes, in which little cooperation is displayed by the 'cheater' but they gain benefit from the mutualistic cooperation displayed by other microbes [23]. Interestingly, cheating behaviour may not only benefit the cheater but also can help maintain biodiversity [24], therefore may play an important role in the stability or resilience of complex ecosystems like the gut of vertebrates.

In order to understand these temporal ecological interactions at the plant-microbiome interface in the rumen, we used prokaryotic metatranscriptomics and gene network analysis of the attached microbial community on fresh perennial ryegrass (PRG) incubated in sacco in the rumen over an 8 - $h$ period. For the first time, we provide in-depth, gene expression-based understanding of the ecological interactions governing temporal niche specialisation, cooperation and competition within the PRGattached rumen microbiome. This knowledge is essential for understanding microbial drivers of energy-harvesting capacity, which influence ruminant feed efficiency and emissions, as well as facilitating breeding of improved PRG cultivars for ruminant livestock.

\section{Methods \\ Growth and preparation of plant material for in sacco incubations}

PRG (Lolium perenne cv. AberDart) was grown from seed in plastic seed trays (length $38 \mathrm{~cm} \times$ width $24 \mathrm{~cm} \times$ depth $5 \mathrm{~cm}$ ) filled with soil/compost (Levington's General Purpose). Plants were kept in a greenhouse under natural light with additional illumination provided (minimum 8-h photoperiod). Temperature was controlled (22/ $19{ }^{\circ} \mathrm{C}$ day/night) and plants were watered twice a week. Plants were harvested after 6 weeks by cutting them (approx. $3 \mathrm{~cm}$ above soil level) directly before use, and then further processing them into $1 \mathrm{~cm}$ sections using scissors. Triplicate samples of harvested plant material were also snap frozen in dry ice and stored at $-80{ }^{\circ} \mathrm{C}$ for profiling of plant epiphytic prokaryotes (0-h samples).

\section{Ruminal in sacco incubations}

Three mature, rumen-cannulated, non-lactating Holstein $\times$ Friesian cows were used for this experiment. The experiment was conducted with the authority of licences under the United Kingdom Animal Scientific Procedures Act, 1986, and managed according to the protocols approved by the Aberystwyth University Animal Welfare and Ethics Review. For 2 weeks prior to the experiment, cows were fed a diet of straw and grass silage ad libitum $\left(\sim 6.5 \mathrm{~kg}\right.$ dry matter day $\left.^{-1}\right)$ and were permitted field grazing on a permanent ryegrass pasture for at least $4 \mathrm{~h}$ / day. For the duration of the experiment, animals were fed silage daily in two equal meals at 07:00 and 16:00 and had constant access to straw when not field grazing.

The nylon bag technique was used as described previously $[25,26]$. Stitched nylon bags $(10 \mathrm{~cm} \times 20 \mathrm{~cm})$ of 
$100-\mu \mathrm{m}^{2}$ pore size were filled with $15 \mathrm{~g}$ (fresh weight) of the processed 1-cm length PRG and sealed at all perimeters by heating (Impulse sealer, American Int, Nl Electric, USA). For each cow, 10 bags were then connected to a $55-\mathrm{cm}$ plastic-coated flexible cable with lacing cords and then placed in the rumen before being attached to the cap of the fistula. Bags were placed simultaneously in the rumen of each cow shortly after animals were offered their first silage meal of the day, and two bags were removed after 1, 2, 4, 6 and $8 \mathrm{~h}$ of incubation then processed by washing with distilled water $(500 \mathrm{~mL}$ added to plant material within bags and bags gently squeezed thereafter) to remove loosely attached microbes followed by immediate freezing in dry ice and storage at $-80{ }^{\circ} \mathrm{C}$ until RNA extraction.

\section{RNA extraction}

Frozen samples were ground to a fine powder under liquid nitrogen and then RNA was extracted using a hot phenol method [27]. Essentially, aquaphenol $(10 \mathrm{~mL})$ was added to the ground sample and then the sample was incubated at $65{ }^{\circ} \mathrm{C}$ for $1 \mathrm{~h}$. Tubes were inverted before chloroform was added $(5 \mathrm{~mL})$. Tubes were centrifuged $\left(5,000 \times g\right.$ for $30 \mathrm{~min}$ at $\left.20^{\circ} \mathrm{C}\right)$ and then the upper phase was removed, then the procedure was repeated by the addition of more chloroform $(5 \mathrm{~mL})$ and centrifugation. Lithium chloride ( $2 \mathrm{M}$ final concentration) was then added, to remove any contaminating DNA, and samples stored overnight at $4{ }^{\circ} \mathrm{C}$. Samples were subsequently centrifuged $\left(13,000 \times g\right.$ for $30 \mathrm{~min}$ at $\left.4{ }^{\circ} \mathrm{C}\right)$ and the supernatant discarded, then the procedure was repeated to ensure all DNA was removed. Once the supernatant was discarded the pellet was resuspended in ice-cold $80 \%$ ethanol and centrifuged $\left(13,000 \times g\right.$ for $15 \mathrm{~min}$ at $\left.4{ }^{\circ} \mathrm{C}\right)$, this was repeated twice before the pellet was air-dried and resuspended in molecular-grade water. The absence of DNA in all samples was confirmed using $16 \mathrm{~S}$ rDNA PCR using non-barcoded primers and subsequent agarose gel electrophoresis as described in Huws et al., [10]. The quality and quantity of retrieved RNA was assessed using the Experion automated electrophoresis system and a RNA StdSens Analysis kit (Bio-Rad Ltd., UK).

\section{rRNA removal and metatranscriptome sequencing}

Prokaryotic mRNA was enriched in all samples by firstly removing the polyA fraction of the mRNA pool using a MicroPoly(A)Purist kit (Ambion) according to the manufacturer's protocol. Eukaryotic 18S rRNA was then minimised using both the RiboMinus plant and eukaryote kits (Invitrogen, Carlsbad, USA) according to the manufacturer's protocols. Finally, 16S rRNA was minimised using the Ribo-Zero rRNA removal kit for bacteria (Epicentre, Madison, USA) according to the manufacturer's protocol. The resulting enriched prokaryotic mRNA was prepared for sequencing using the TruSeq stranded mRNA library prep kit (Illumina, California, USA) following the manufacturer's guidelines. Subsequently, library sequencing was completed using the Illumina HiSeq 2500 (Illumina, California, USA) and a 100-bp paired-end sequencing approach.

\section{Metatranscriptome assembly}

A flow diagram showing the steps taken to prepare and analyse the sequences obtained is shown in Supplementary Figure 1. Essentially, the assembly was performed through a series of steps in order to reduce the complexity of the process. First, the quality of raw reads was evaluated with FastQC [28] version 0.10.1, and subsequently trimmed using Trimmomatic [29] by 9 base pairs at the $5^{\prime}$ end and 3 base pairs at the $3^{\prime}$ end, including remaining adapters. Following this step, the removal of any remaining rRNA contamination was performed using MGKit (script rRNA-protozoa.py [30];) and a custom database of rRNA sequences built by retrieving (i) the RDP databases for archaea, bacteria and fungi [31], release 11_2 and (ii) rRNA genes from protozoan species from the EBI-ENA, This custom database was used with bowtie2 (2.1.0) [32] using the '--no-mixed --local --sensitive-local $-\mathrm{N} 1$ ' options, saving only the unaligned and hence non-rRNA reads (using the '--un-conc-gz' option) to files. The retained reads were then aligned to the draft Lolium perenne genome [33] using bowtie 2 and the same settings, retaining only those reads that did not align to the genome. To utilise the availability of fully sequenced prokaryotic genomes from cultured organisms, all reads that aligned (using the aforementioned options) to a collection of 246 publicly available genomes, in particular from the Hungate1000 collection [34] (list of genomes available in Supplementary Excel 1), were not included in for de novo assembly and instead were retained for later inclusion in the expression quantification stage.

The first step of the meta-transcriptome assembly of non-aligned reads involved digital normalisation to reduce the bias from more abundant transcripts in the samples. The khmer package (version 2.1.2) [35] was used in a two-step procedure. First, the reads were normalised on a per-sample basis using the normalise-bymedian.py script (with the options -p, $-\mathrm{k} 20,-\mathrm{N} 4,-\mathrm{x}$ 16e9, -C 20). Afterwards, a collective normalisation was carried out pooling all per-sample normalised reads. The same parameters as the per-sample normalisation were used, except for - $\mathrm{x}$ (an option to define the hash table size), which was set to $32 \mathrm{e} 9$ to account for the larger size of the input data. Following digital normalisation, an overall assembly was carried out using velvet version 1.2.10 [36] with a kmer size of 31 and automatic expected coverage (-exp_cov auto). Finally, alignments of 
all the reads used in the assembly to the final resulting assembly were created using bowtie2 (2.1.0) [32] using the '--no-mixed --local --sensitive-local -N 1'. The resulting alignments of each sample to the assembly were combined with the alignments of each sample to the cultured genomes (from the earlier step) for expression quantification.

\section{CDS prediction and quantification}

Putative coding sequences (CDS) were predicted in the de novo assembled transcriptome using the default options of the 'TransDecoder.LongOrfs' script from Transdecoder: https://github.com/jls943/TransDecoder [37, 38] which identifies ORFs that are at least 100 amino acids long. These were combined with the predicted CDS from the genomes that had aligned mRNA reads. These annotations formed the set of CDS which were used in the subsequent functional and taxonomic analyses. Abundance (counts) of reads mapping to each CDS annotation was calculated with htseq-count from the HTSeq package [39] (options: -s no; -t CDS; -q). The predicted amino acid (AA) sequences of these CDS were used in the subsequent annotation steps.

\section{Taxonomic annotation}

The blastp command of the BLAST package (10.1186/ 1471-2105-10-421) was used with the -outfmt 6 option against the NCBI nr database. The output was then passed to the add-gff-info script of the MGKit package, which uses a last common ancestor algorithm in the taxonomy command to resolve the taxon identifiers of the annotations. The options used with add-gff-info taxonomy were as follows: -s 40 in order to use only BLAST results that have a bit score of at least 40, -1 to use the last common ancestor algorithm and -a 10 to use only the results that are within 10 bits from the maximum bit score for each annotation. For the CDS from the genomes, the resulting predicted taxonomic assignments were cross-checked against the species the genomes represented in order to provide validity for the de novo predictions for the CDS from the assembly.

\section{Gene family identification}

A series of hierarchical clustering approaches were used to identify gene family clusters within and across species. Firstly, an all-against-all search of all the AA sequences was carried out using Diamond [40], where the maximum number of target sequences was set to $1,000,000$ with a minimum bit score of 60 and all other options set to the default. Next, EGN was used to identify an initial round of gene similarity clusters using the Diamond allagainst-all search results as input with the 'gene network' option and the following settings: E-value threshold $=1 \mathrm{e}-05$, hit identity threshold $=20 \%$, identities must correspond at least to $20 \%$ of the smallest homologue, no best reciprocal condition and no hit coverage condition enforced [41]. Secondly, all the AA sequences were used as input to the EGGNOG mapper (v1) [42] to generate (where possible) KEGG ortholog (KO) IDs for the sequences [43-46]. The KOs were then used to identify where different EGN gene clusters were recognised as having the same function, allowing them to be combined into higher level functional clusters using igraph in $\mathrm{R}$ [47]. This was followed by further manual refinement of the functional groups in MS Excel using the information from the EGGNOG annotations of the AA sequences. Finally, the taxonomic assignment, functional cluster membership and expression (count) information across each of the samples for every predicted CDS was combined into an overall table for subsequent analyses.

\section{Network analysis}

To perform the co-expression network analysis at the family taxonomic level, the count data needed to be preprocessed. The taxonomic families with less than 10 genes expressed were removed from the dataset and the sum of the expression for all genes in the remaining taxonomic families was calculated. Next, to account for differences in sequencing depths in each sample, the summed count data were scaled by dividing each value by the sum of the total counts from the sample it belonged and then multiplying them by the median of all sample sums. Then, the scaled data were further filtered using variable filtering based on inter-quartile range (IQR; $[48,49]$ ), where taxonomic families with expression lower than the 1st quartile (25th percentile) were removed. The final stage of data pre-processing was data normalisation, which was completed using regularised $\log$ transformation [50].

Co-occurrence networks were subsequently constructed based on the results of correlation analysis. The correlation analysis was conducted with Spearman's rho rank correlation and the results were filtered based on both correlation coefficients and their corrected pvalues. Only the interactions with a correlation coefficient larger than 0.7 and (Benjamini-Hochberg) adjusted p-values less than 0.1 were used for the network analysis. The constructed network was then explored and visualised using the open-source software Gephi [51]. Modularity metrics were calculated in Gephi to detect the clusters in the constructed network.

Putative keystone taxa identification was performed within each cluster of the network [52]. We hypothesised that keystone taxa would have a large impact on the community network, and any absences of them should lead to major disruption to the network [53]. To describe the importance of keystone taxa, we used a set of network-level measures: transitivity, density, 
modularity, average path length and centralisation of eigenvector. Transitivity measures the probability of the adjacent vertices. The graph's density calculates how many edges are compared to the maximum possible number of edges between vertices. Modularity is designed to measure the strength of division of a network into modules. The average path length is calculated as the shortest paths between all pairs of vertices. Eigenvector centralisation measures from the centrality scores according to the eigenvector centrality of vertices. In order to determine the keystone taxa, we calculated these measurements with the full network and then again after removing a node. This was calculated for every node in the network. Thus, the differences in the measurements between each removal network and the complete network could be used to reveal those taxa whose removal had the largest detrimental effect on the community network structure and, therefore, could be identified as putative keystone taxa. Based on the mechanism of these measures, if one node has a large impact on the cluster, the differential values of transitivity and density should be reduced whilst modularity, average path length and centralisation of eigenvector should be increased. For each measure, we ranked each node based on the difference calculated for these values following the node removal and further produced an overall ranking for each node using the Borda count method (sum of ranks), which is widely used in the voting method for decision-making of multiple ranks. The overall topranked families from the Borda count were identified as putative keystone taxa. To fulfil this task, we used the $\mathrm{R}$ package igraph [47]; the scripts and data used to carry out the analyses described above are available on the Open Science Framework at https://osf.io/rx9h2/.

\section{Temporal gene expression analysis}

In order to carry out the statistical analysis of gene expression, the expression information was summarised by taxonomic family and functional cluster (generated as earlier described). This resulted in raw count data for each timepoint from each functional cluster expressed in every taxonomic family [54]. This data was used as input to the Bioconductor package DESEQ2 in R [50]. In this analysis, the design was 'cows+time'. Initial testing of the effect of cows and time was carried out using the likelihood ratio test 'LRT' in DESEQ2, where first the effect of 'cows+time' was tested against a reduced model of 'time', testing for any interactions with the cows the samples were taken from. Secondly the model 'cow+ time' was tested against a reduced model of 'cows' to test the effect of time. Only those genes identified to have a significant interaction with time from the LRT (and excluding the 2 genes that had an interaction with cows) were retained for subsequent pairwise differential expression analysis. All 10 possible pairwise comparisons between the 5 sampled timepoints (excluding timepoint zero) was carried out to identify the pattern of differential expression (DE) for those genes with a significant interaction with time using a Benjamini-Hochberg padjusted cut-off of 0.1 to identify significance.

Finally, 'significance groups' across time (analogous to the output from Tukey's HSD test) were identified for each gene in each taxonomic family to allow identification of patterns of DE over time and to allow clustering of genes by their shared DE pattern. This was carried out in $\mathrm{R}$, using igraph, to identify for each gene in each taxonomic family the maximal cliques in the network of timepoints that were not significantly different to each other. These maximal cliques were then converted into labels for identifying the significance groups in subsequent visualisations. The $\mathrm{R}$ script and data used to carry out the temporal gene expression as described above is available in the Open Science Framework at https://osf. io/rx9h2/.

\section{Detailed annotation and temporal abundance of glycosyl hydrolase, peptidase and quorum sensing genes}

The functional clustering described earlier grouped all glycosyl hydrolases $(\mathrm{GH})$ and peptidase genes into single clusters containing all variants of these enzymes. In order to facilitate a further in-depth analysis of the expression of these important enzymes, a more detailed functional analysis of these two enzyme groups was carried out. Separation of GH families was carried out in reference to the annotations available in the CAZy database (http://www.cazy.org/; [55]) using DBCan (version 2.0.0) [56], Separation of the peptidases families was carried out in reference to the MEROPS [57] database using Clustal Omega (version 1.2.4) [58] to identify groups of peptidase families in the database which were then assembled into profiles using HMMER (version 3.3) [59]. These profiles were then used to assign peptidases to families using HMMER. To facilitate the statistical analysis of this subset of the dataset, the sum of the expression of all genes assigned to each $\mathrm{GH}$ and peptidase family was calculated for each taxonomic family.

For the quorum-sensing (QS) genes, the functional clustering identified a cluster of S-ribosylhomocysteine lyase (LuxS) genes, involved in Auto-inducer-2 (AI-2) based QS, from 5 different taxonomic families. However, no clusters of $\mathrm{N}$-acyl homoserine lactone (AHL) were identified, so no analysis could be undertaken for this gene. Next, the expression of each of the GH, peptidase and QS families was normalised by calculating the transcripts per million (TPM) for every gene in the entire transcriptome in MS Excel and then extracting the calculated values for the members of the $\mathrm{GH}$, peptidase 
and QS families. This commonly used normalisation method for RNAseq data utilises the knowledge of the length of each gene and the total sequencing effort and provides an estimate of the number of RNA molecules from this gene for every million RNA molecules in the sample. The GH and peptidase families were then separately analysed as follows. First, the TPM for all genes identified as belonging to the same enzyme family were summed for statistical analysis. For the GH and peptidase families, any that fell outside the $95 \%$ most highly expressed across all timepoints for that type (i.e. GH or peptidase) were excluded. , for each $\mathrm{GH}$ and peptidase family, an analysis of variance (ANOVA) was performed on the summed TPM values in order to identify any significant interactions with time, including cow as a fixed effect in the design (with the 'aov' command in R). For the QS genes, the TPM values from each taxonomic family were summed and an ANOVA was performed to identify any significant interactions of the expression of the QS genes in each taxonomic family with time. All the ANOVA p-values were then corrected for multiple testing using the Benjamini-Hochberg method with a value of $<0.1$ used to determine enzyme families with a significant interaction with time. For each of these with a corrected p-value $<0.1$, Tukey's HSD test was performed (using the 'TukeyHSD' command in R) to identify where the significant differences were occurring and to assign significance groupings across timepoints. The contribution of each taxonomic family to the expression of each GH and peptidase family at each timepoint was also determined. All data was visualised using "ggplot2" in R. Detailed isoform expression analysis of GH families $3,5,9,10$ and 48 was carried out by extracting the AA sequences for each separately and aligning with default options in Muscle (v3.8.1551 [60];) followed by phylogenetic tree reconstruction using RAxM-ng (v1.1.0 [61];) using a JTT+G model. Finally, the expression profiles of each isoform at each timepoint were mapped to the resulting phylogenetic tree using iTOL [62]. The $R$ scripts used to carry out this analysis are available in the Open Science Framework at https://osf.io/rx9h2/.

\section{Results}

\section{Overview of sequencing data}

We generated prokaryotic metatranscriptome data for PRG-attached prokaryotes incubated over time (1, 2, 4, 6 and $8 \mathrm{~h}$ ) within the rumen of three cannulated Holstein $\times$ Friesian cows (two replicates/animal/timepoint with replicates within animals being pooled pre-sequencing) and the PRG attached bacteria at $0 \mathrm{~h}$ (three $0 \mathrm{~h}$ preincubation PRG RNA extractions being pooled presequencing). Therefore, a total of 16 samples (i.e. 3 cows $\times 5$ timepoints +0 -h sample) were sequenced to assess the gene expression of PRG attached prokaryotes over time. Information on the number of reads generated, pre and post filtering, alignment to other datasets and the number of genes expressed, for each sample can be found in Supplementary Table 1. Average read abundance/sample was $14,825,273$. However, 0 -h sample reads were low at 2,060,217 and did not align to any of the Hungate genomes due to the fact that bacteria colonising PRG preincubation were epiphytic in nature (Supplementary Excel 1). Taxonomic families associated with this timepoint further decreased post-rumen incubation; therefore, these were not included in the subsequent metatranscriptome analysis (Supplementary Excel 1).

\section{Network analysis}

Family-based gene network correlations, showing both positive and negative gene correlations are shown in Fig. 1. The layout of the network is ForceAtlas2 [63] where connections or edges are presented that have correlation coefficients (Spearman's rho) larger than 0.7 and adjusted pvalues less than 0.1 . The sizes of the nodes are proportional to the expression of genes from the corresponding family across all timepoints. The size of the nodes indicates that (in descending order) Lachnospiraceae, Prevotellaceae, Ruminococcaceae, Selemonadaceae, Spirochaetaceae, Methanobacteriaceae, Fibrobacteraceae and Eubacteriacaeae dominate, irrespective of incubation time. The family Lachnospiraceae was dominated by two genera (Butyrivibrio and Pseudobutyrivibrio), whilst the families Prevotellaceae, Ruminococcaceae, Selemonadaceae, Spirochaetaceae, Methanobacteriaceae, Fibrobacteraceae and Eubacteriacaeae were each comprised of a single genus only, namely: Prevotella, Ruminococcus, Selenomonas, Treponema, Methanobrevibacter, Fibrobacter, and Eubacterium respectively (Supplementary Excel 1). In the interest of not repeating family and genera in every instance, we refer mainly to families in the results presented below. Further information for genera within these and other families can be found in Supplementary File 1.

The network analysis also shows that there are two large clusters in the network with strong positive correlations, and these clusters are separated by red lines which indicate negative gene expression-based correlations between the two large clusters (Fig. 1). These two clusters predominantly represent primary $(\leq 4 \mathrm{~h}$ post rumen incubation) and secondary ( $\geq 4 \mathrm{~h}$ post rumen incubation) colonisation phases and can be considered two sub-microbiomes (Fig. 1). The primary sub-microbiome is largely dominated by Prevotellaceae, whilst the secondary sub-microbiome is dominated by Lachnospiraceae, and to a lesser extent Ruminococcaceae and Eubacteriaceae. A further group of bacterial families are independent of the primary and secondary submicrobiomes, namely families Selemonadaceae, Spirochaetaceae, Methanobacteriaceae, Bifidobacteriaceae, 


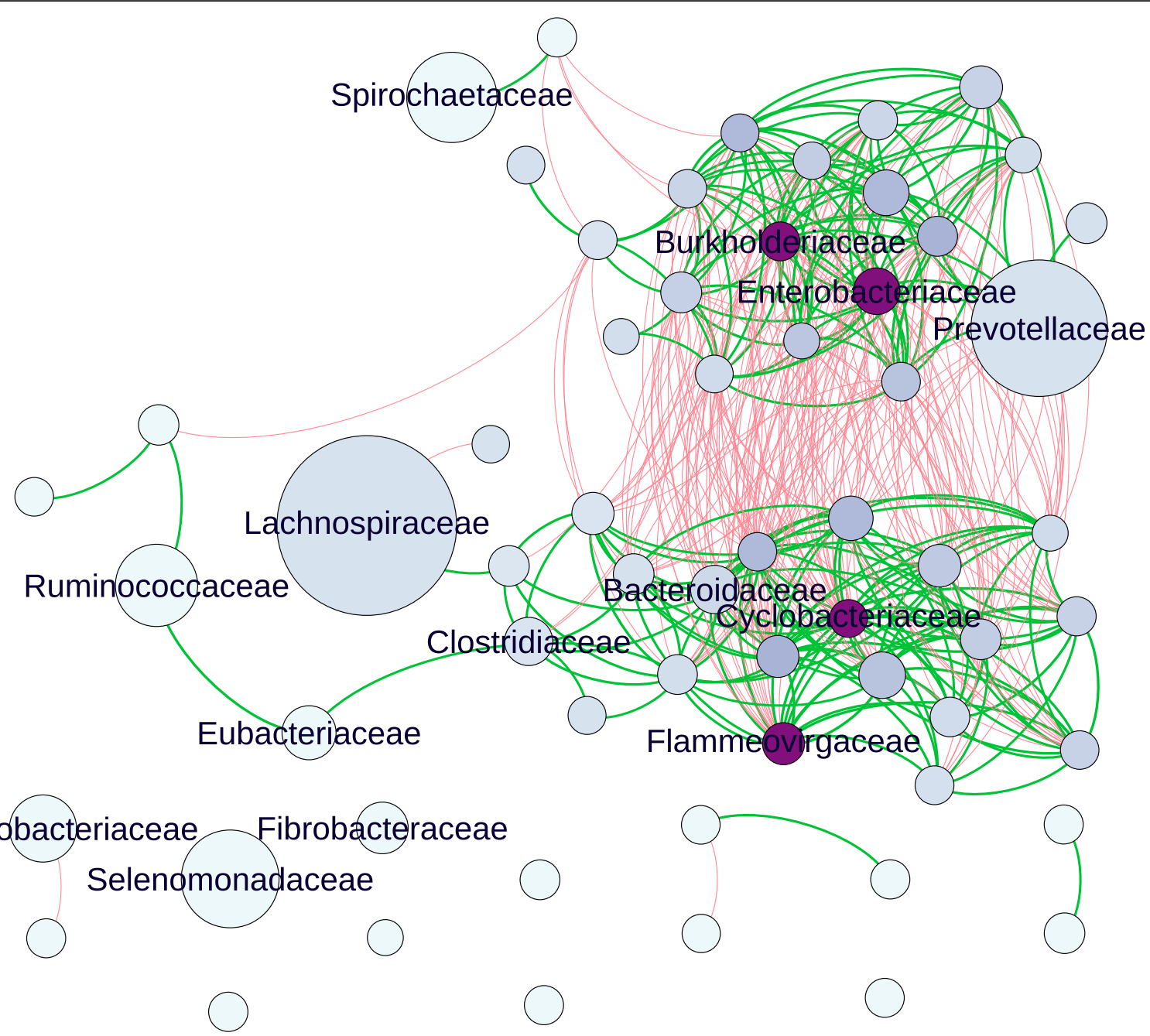

Fig. 1 Co-occurrence gene network map showing positive (green lines) and negative gene correlations (red lines) for genes assigned to families. The size of the node denotes family relative abundance. Nodes in purple indicate putative keystone families and the gradient in colours relates the ranking of the families as 'keystone', where the darkest were the top ranked. The top cluster relates to primary colonisation $(<4 \mathrm{~h})$ and the bottom secondary colonisation ( $>4 \mathrm{~h}$ ) events. Names of dominant and keystone familes are shown only due to the complexity of the network, for information on all interactions refer to Supplementary Tables 2 and 3

Fibrobacteraceae, Lactobacilliaceae, Enterococcaceae, Pseudomonadaceae, Phyllobacteraceae, Streptomycaceae, Neisseriaceae, Nocardiodiaceae, Commomonadaceae, Desulfovibrionaceae and Pasturellaceae (in descending order of relative abundance). Also, of particular note is the finding that the dominant families had few or even no gene correlation, suggesting selfish non-cooperative behaviour, although Prevotellaceae was an exception (Fig. 1).

To identify keystone taxa, a set of node measurements were calculated. Amongst these measurements, five centrality metrics (transitivity, density, modularity, average path length and centralisation of eigenvector) were also used to identify putative keystone taxa as previously done by others [53, 64-66]. These measurements included five centrality metrics: transitivity, density, modularity, average path length and centralisation of eigenvector. For the primary sub-microbiome the highest-ranking putative keystone families were Burkholderiaceae and Enterobacteriaceae, and for secondary sub-microbiome Cyclobacteriaeceae and Flammeovirigaceae (Fig. 1; Supplementary Tables 2 \& 3). This suggests these families have an important role in the cohesion of these submicrobiomes although they have a much lower activity than the dominant families and are within the lower $10 \%$ of those reported in Supplementary Fig. 2A \& $\mathrm{B}$, and hence are not directly shown within the graph.

\section{Temporal niche specialisation}

A total of 1513 genes were differentially expressed (DE) by the prokaryotes colonising PRG in the rumen over time (these values exclude genes lower than $10 \%$ of total 
expression levels) (Fig. 2A). These genes were representative of all general functions, including cellular processing, information storage and processing, metabolism, and others which were poorly characterised or unknown (Fig. 2B). Twenty-eight distinct DE patterns across all five timepoints were observed in these genes, with as few as three genes and up to as many as 170 genes showing any particular DE pattern (Fig. 2). The DE pattern with the highest total expression across all timepoints consisted of 69 genes and was expressed in higher abundance during secondary colonisation $(\geq 4 \mathrm{~h}$ rumen incubation) compared to primary colonisation $(\leq 4 \mathrm{~h}$ rumen incubation) (Fig. $2 \mathrm{C}$ and D). These 69 genes were mainly expressed by Lachnospiraceae, with lower expression also evident within the Prevotellaceae (Fig. $2 \mathrm{E})$. Interestingly, the DE pattern with the secondhighest expression (consisting of 96 genes) showed the opposite pattern (i.e. higher abundance during primary colonisation) and was dominated by Prevotellaceae, consistent with the network analysis. The remaining DE patterns identify further timepoint and taxa-specific expression profiles worthy of further in-depth analysis. Summarising these DE patterns by function identified many bacterial families with time-point specific functional roles (Figs. 3, 4, 5, 6, 7 and 8; Supplementary Figs 3-32). As there are too many temporal functional changes evident within this complex microbiome to outline all in detail here, we have provided the raw data and graphical representation of the taxonomy by temporal expression patterns for all Enzyme Commission (EC) categories in the supplementary material (Supplementary Figs 3-32) for those with an interest in specific microbial functions. In this study, we concentrated on genes involved in methane metabolism, protein and carbohydrate breakdown and quorum sensing.

Genes involved in methane metabolism were mainly expressed by Lachnospiraceae (interestingly mainly by genus Butyrivibrio and not Pseudobutyrivibrio) and Methanobacteriaceae, with expression increasing during secondary colonisation $(\geq 4 \mathrm{~h}$ rumen incubation; Fig. 3; Supplementary Excel 1). Given that Butyrivibrio dominated the expression profile (Supplementary Excel 1) and they produce butyrate and hydrogen from fermentation of complex and simple carbohydrates [67], it is likely that the Methanobrevibacter, which utilise hydrogen to produce methane, increase in response to the higher hydrogen levels allowing them to increase methane formation and release as

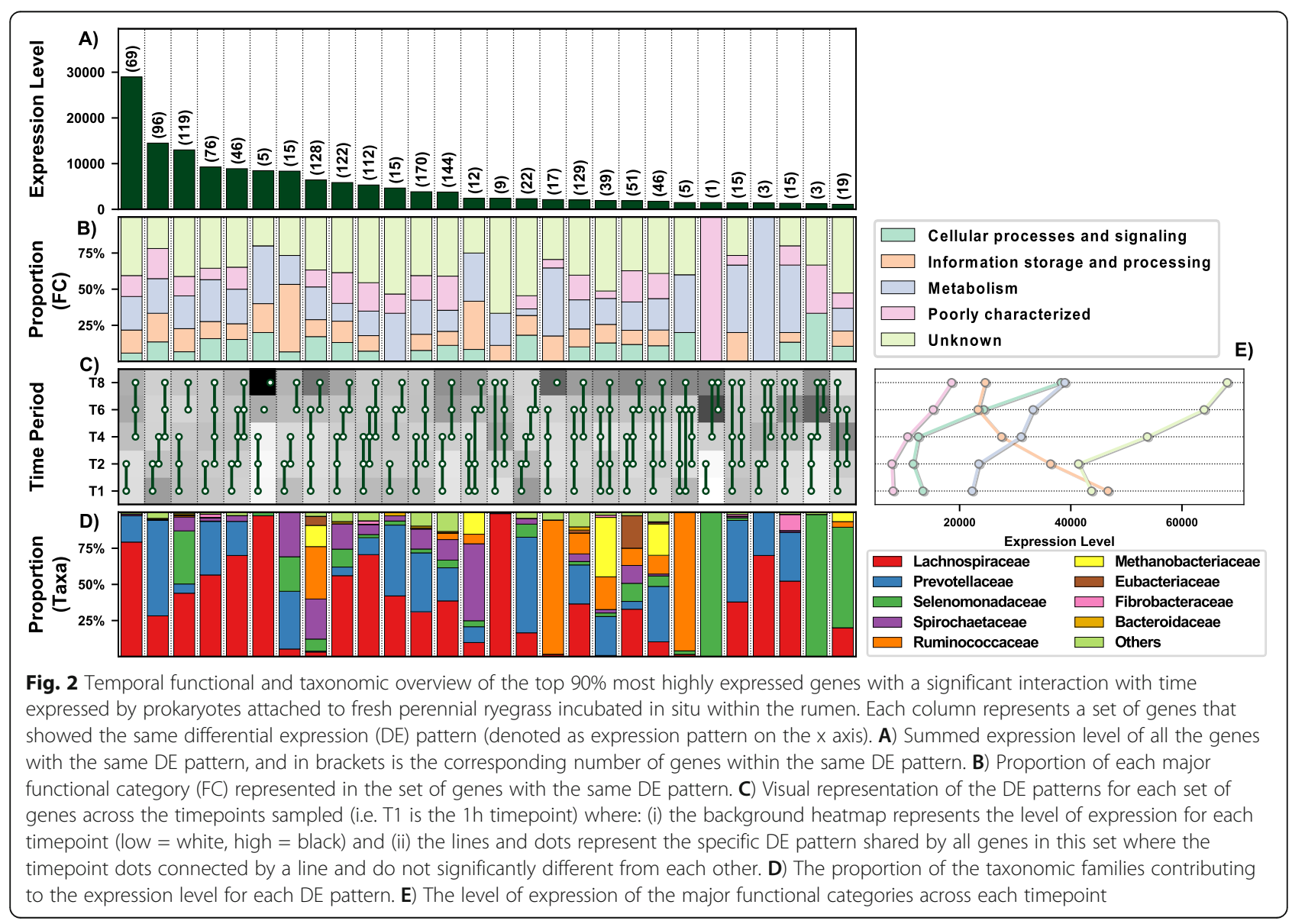




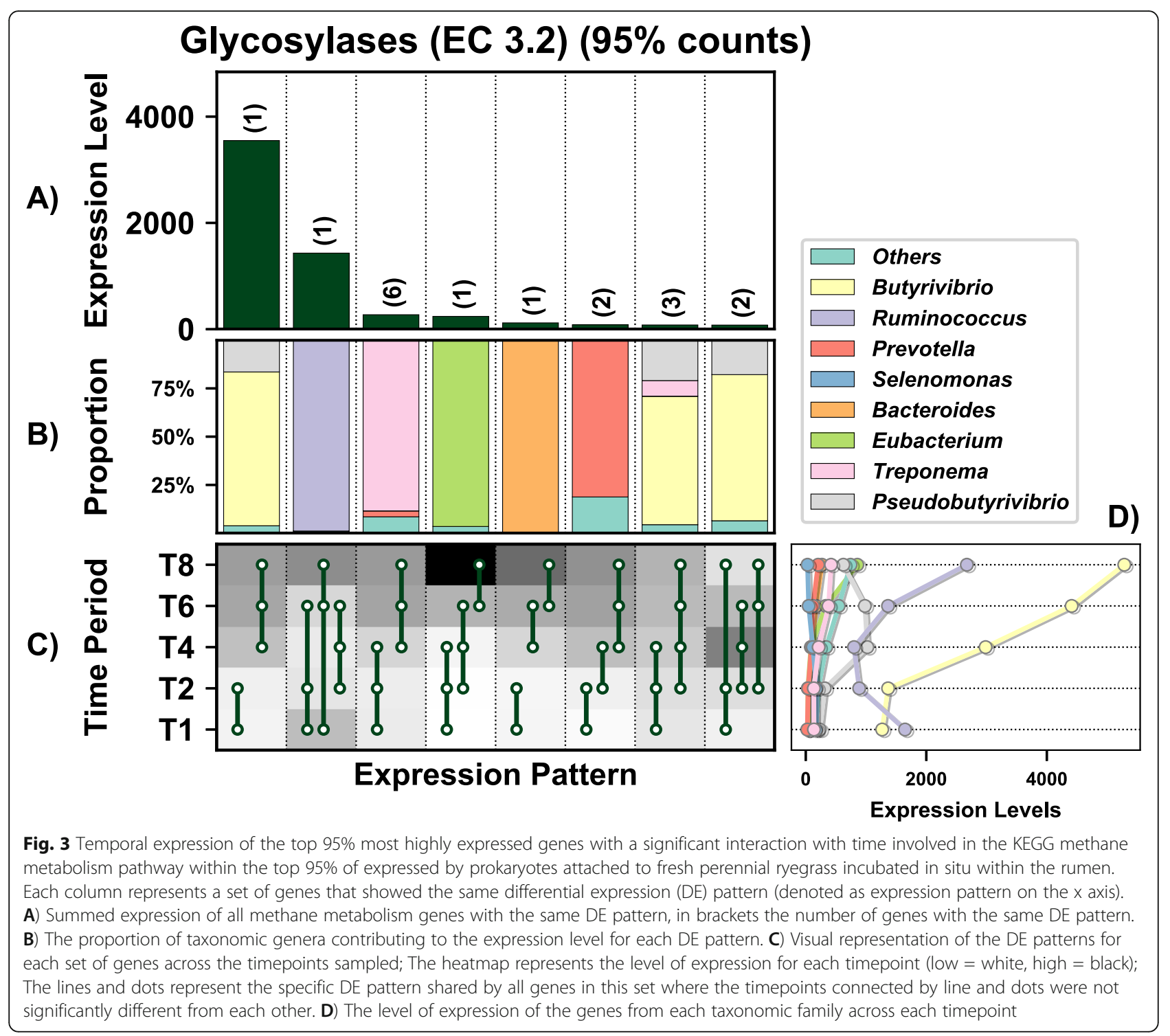

supported by KEGG information (Supplementary Figure 33).

Peptidase genes are important to the host in terms of breaking down protein. In total 7 genes encoding peptidases (EC 3.4) were detected and these were generally more highly expressed during primary colonisation compared with secondary colonisation, in particular within the family Prevotellaceae, which specialise in this activity (Fig. 4; Supplementary Excel 1). When MEROPS was used for a more detailed analysis of peptidase family expression (Supplementary Excel 1; Fig. 4) this indicated that 38 peptidase families were within the top $95 \%$ of all expression of this type of gene (Supplementary excel 1; Supplementary Figure 34). These genes families were most predominantly expressed by: M50, Ruminococcaceae; M24b, Lachnospiraceae; s01c, Prevotellaceae and Ruminococaceae; M16b, Prevotellaceae (Supplementary
Excel 1; Supplementary Figure 34). In terms of temporal changes in peptidase expression, significant changes over time were noted only for M24b and M15a (expressed mainly by the bacterial families Selemonadaceae and Ruminococcaceae), with expression increasing during secondary colonisation (Supplementary Excel 1; Supplementary Figure 34). This is the converse to what was seen with overall peptidase (EC 3.4) expression data and a consequence of obtaining more granular data using MEROPS. Further elaboration of their precise role was not possible as the exact peptidase function for most of the peptides could not be identified.

Genes encoding glycosyl hydrolases (often also called carbohydrate-active enzymes; CAZymes) (EC 3.2; 17 in total) were mainly upregulated during secondary colonisation ( $\geq 4 \mathrm{~h}$ rumen incubation), especially within the families Lachnospiraceae and Ruminococcaceae, which 


\section{Acting on peptide bonds (peptidases) (EC 3.4) (95\% counts)}

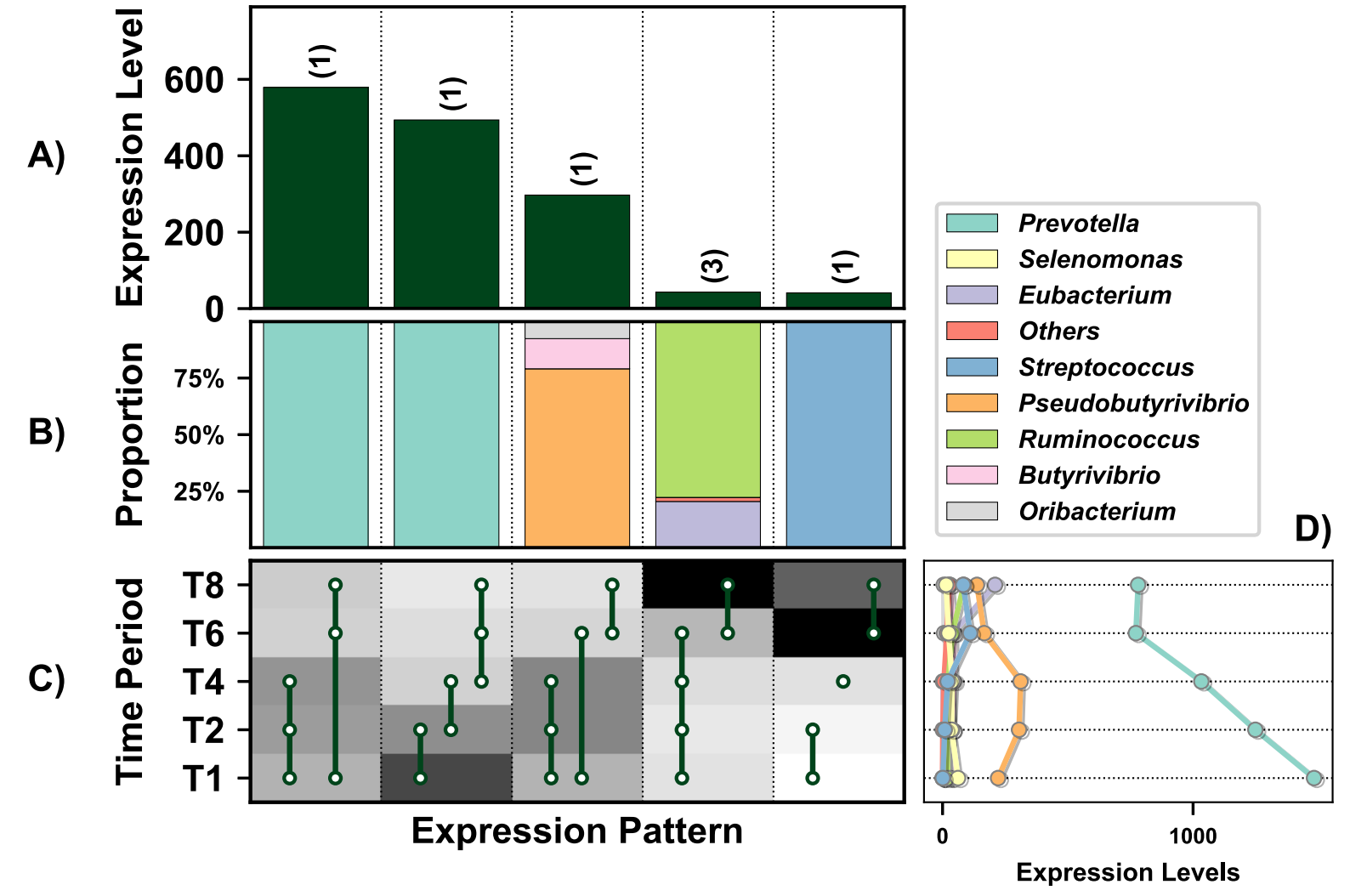

Fig. 4 Overview of the temporal expression of the top 95\% most highly expressed peptidase genes (EC 3.4) with a significant interaction with time expressed by prokaryotes attached to fresh perennial ryegrass incubated in situ within the rumen. Each column represents a set of genes that showed the same differential expression (DE) pattern (denoted as expression pattern on the $x$ axis). A) Summed expression of all genes with the same DE pattern, in brackets the number of genes with the same DE pattern. B) The proportion of taxonomic genera contributing to the expression level for each DE pattern. C) Visual representation of the DE patterns for each set of genes across the timepoints sampled; The heatmap represents the level of expression for each timepoint (low $=$ white, high $=$ black); The lines and dots represent the specific DE pattern shared by all genes in this set where the timepoints connected by line and dots were not significantly different from each other. D) The level of expression of genes across each timepoint

specialise in carbohydrate breakdown (Fig. 6; Supplementary Excel 1). When DBcan was then used to analyse the CAZymes, further resolution was obtained (Supplementary Excel 1; Figure 35). DBcan showed that a total of 18 CAZymes represented $95 \%$ of all expression of this type of gene in the PRG-colonising prokaryotes (Supplementary Figure 35) with enzyme families GH3, 5, 10, 9 and 48 dominating the expression profiles, in descending order (Supplementary Excel 1; Figure 35). Significant changes over time were noted for GH1 ( $\beta$-glucosidases and $\beta$-galactosidases; mainly expressed by Selemonada-

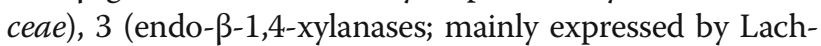
nospiraceae, 5 (endo- $\beta-1,4$-xylanases; mainly expressed by Fibrobacteraceae and Ruminococcaceae), 9 (endo- $\beta-1,4-$ xylanases; mainly expressed by Ruminococcaceae), 10 (endo-endo-beta-1,4-xylanases; mainly expressed by Ruminococcaceae), 11 (endo- $\beta-1,4$-xylanases; Ruminococcaceae), 13 (act on substrates containing $\alpha$-glucoside linkages; Spirochaetaceae and Selemonadaceae), 26 (endo3-1,4-mannanases; Eubacteriaceae, Fibrobacteraceae, Ruminococcaceae and Selemonadaceae) and 57 (includes $\alpha$-amylases, $\alpha$-galactosidases, amylopullulanases and 4$\alpha$-glucanotransferases; Fibrobacteraceae and Prevotellaceae). All of these apart from GH1 increased during secondary colonisation compared to primary colonisation (Fig. 6; Supplementary Excel 1 and Figure 35). In order to contextualise this temporal fresh PRG carbohydrate degradation on a genus basis, a schematic figure was constructed which illustrates the changes in niche specialisation by the rumen bacteria, e.g. GH5 is mainly expressed by Fibrobacter and Ruminococcus during primary colonisation and by Butyrivibrio and Pseudobutyrivibrio during secondary colonisation (Fig. 7). Intriguingly, when these data are examined at an even more detailed level, it is apparent that different isoforms of each of the dominant $\mathrm{GH}$ families (GH3, 


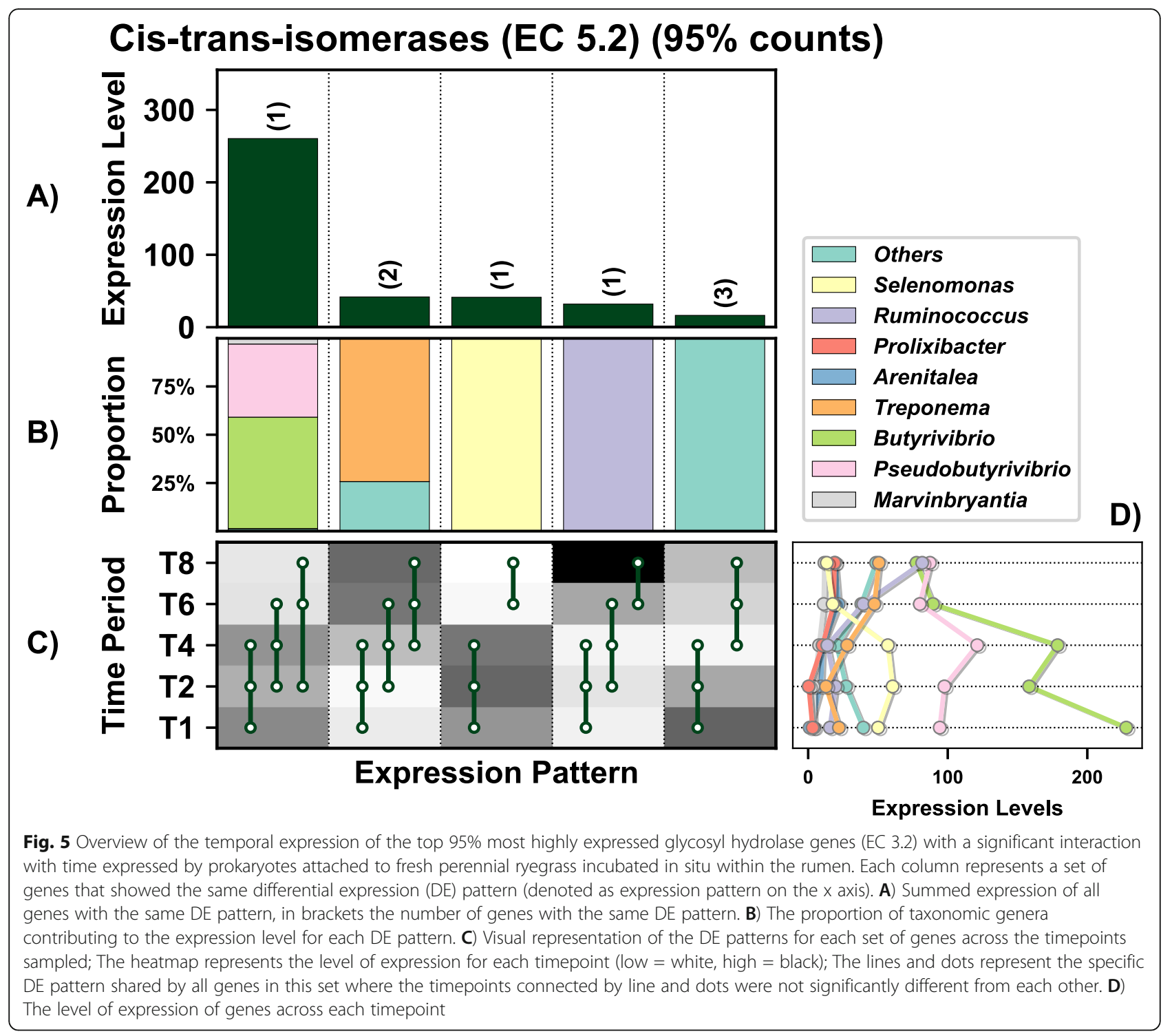

5, 9, 10 and 48) have time-point specific activity (Supplementary Figures 36-40).

To further understand potential drivers of switches from primary to secondary colonisation events, the temporal expression of cell signalling molecules was also investigated. AI-2-based LuxS quorum sensing temporal expression data showed that these genes were mainly expressed by Prevotellacae. It was also evident that Prevotellaceae increased expression of LuxS genes during primary colonisation and thereafter declined, which may partially drive the transition to a secondary submicrobiome (Fig. 8).

\section{Conclusion}

Microbial interactions are complex and often explained as being either positive, neutral or negative [21, 22], with pairs of effects often found, such as in mutualism and competition. Combinations of positive, neutral and negative outcomes can also be found, for example under amensalism and commensalism [21]. This study investigated the breadth of temporal ecological interactions and niche specialisation of fresh PRG-attached rumen prokaryotes. Using metatranscriptomics and network analysis, we show, on a gene expression network basis, that temporal colonisation of fresh PRG involves an array of ecological interactions, with cooperation and mutualism as well as competitive behaviours evident. Primary ( $\leq 4 \mathrm{~h}$ rumen incubation) and secondary $(\geq 4 \mathrm{~h}$ rumen incubation) PRG-attached sub-microbiomes were evident over time, as evidenced by antagonistic negative interactions between the two large clusters. Network analysis also enabled definition of interactions within these sub-microbiomes, including identification of putative keystone families. In terms of key functional 


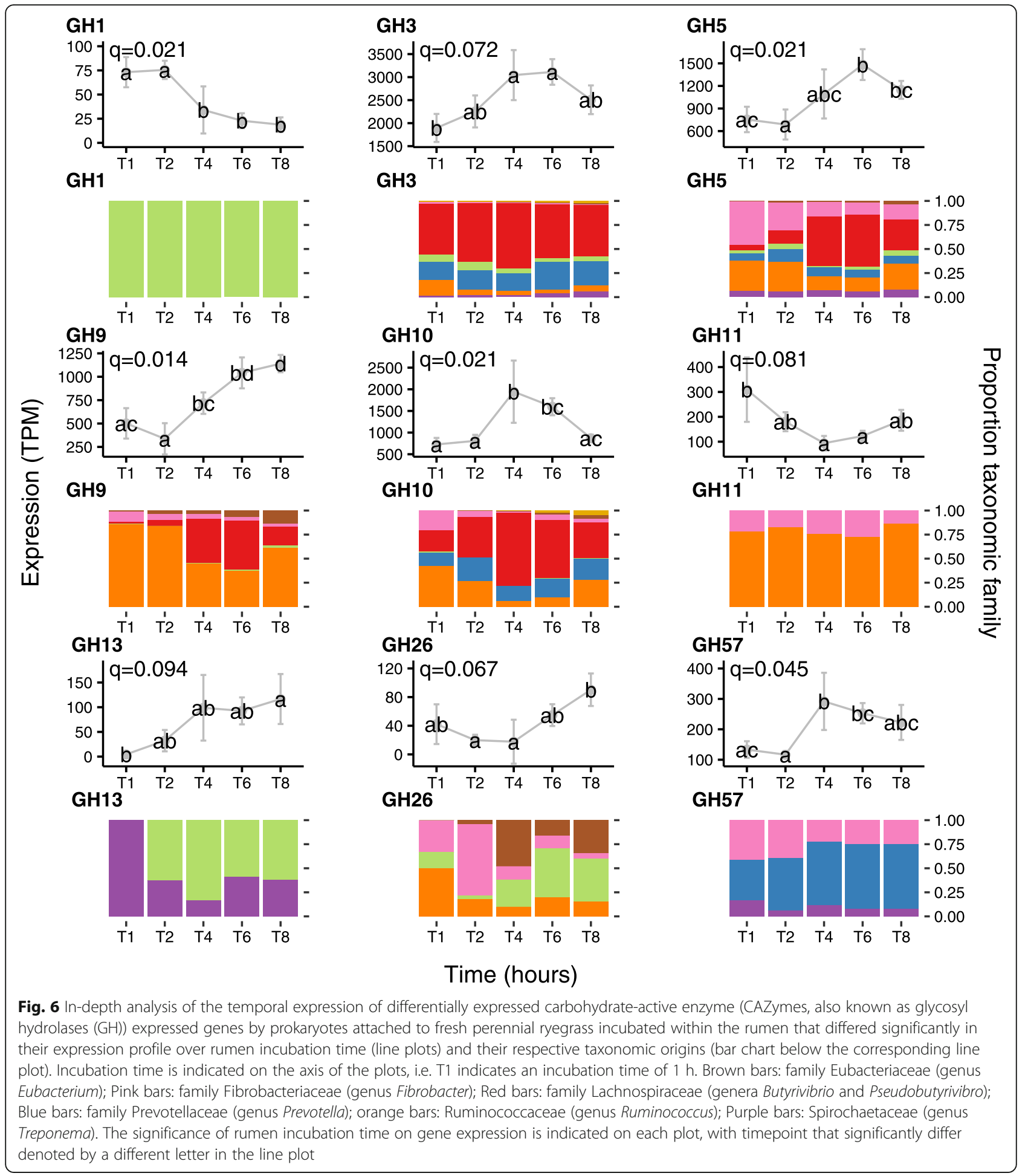

processes for the host, whilst protein breakdown is a continual process with limited differences seen over time and between sub-microbiomes, carbohydrate degradation is higher for many CAZymes during secondary colonisation which is mainly related to the breakdown of complex carbohydrates. Dominant bacteria also commonly change their function over incubation time, presumably because of the need to be ecologically plastic in a changing environment.

In terms of prokaryotic expression, the top 5 families were Prevotellaceae, Lachnospiraceae, Selemondaceae, Ruminococcaeae and Fibrobacteraceae. The family 


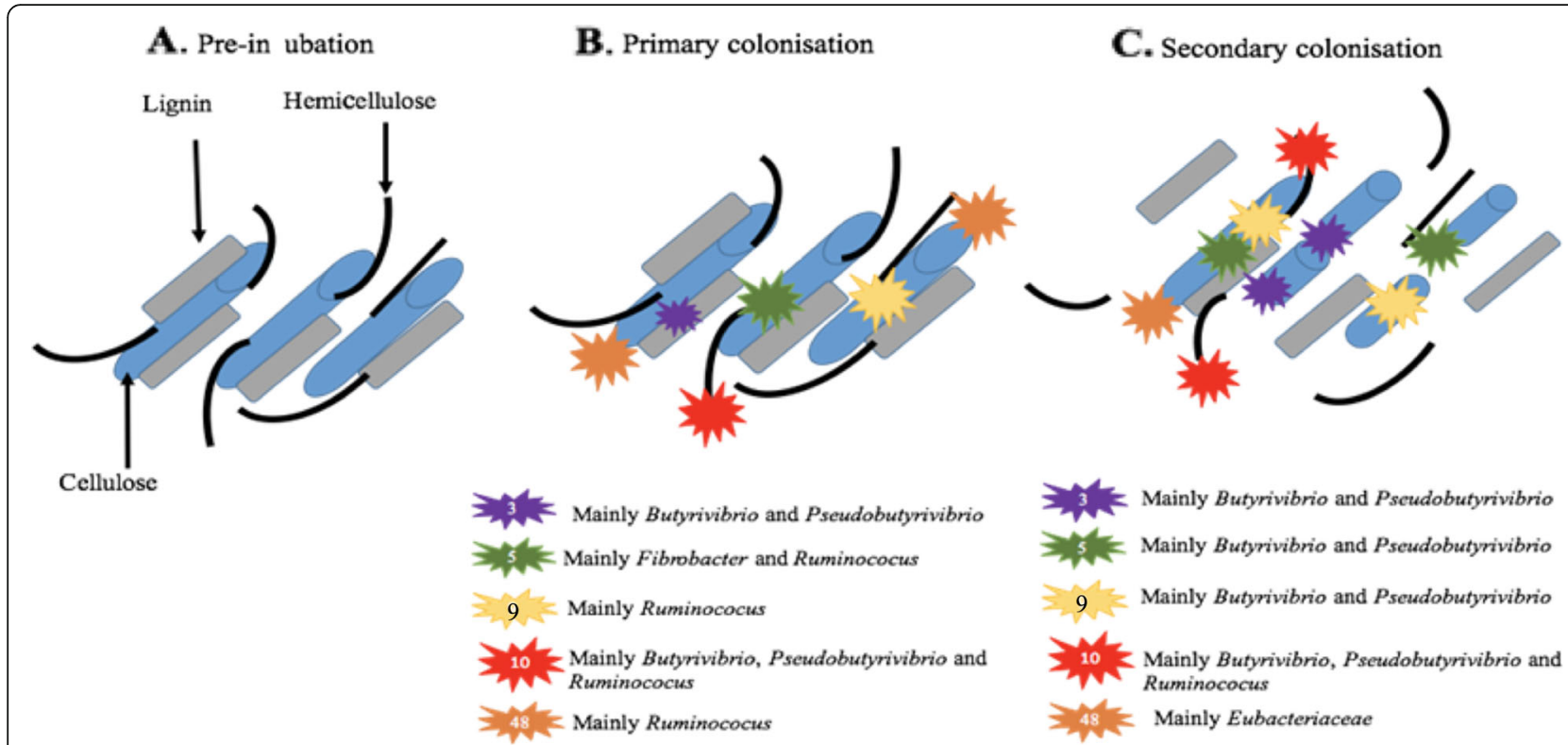

Fig. 7 Diagrammatic representation of fresh perennial ryegrass carbohydrate breakdown over incubation time within the rumen. The diagram illustrates only the dominant carbohydrate-active enzymes (CAZymes, also known as glycosyl hydrolases (GH)).GH family numbers are shown by the numbers in the symbol key. Taxonomic origins of the expressed CAZymes are also shown in the key next to the corresponding symbol. The number of GH symbols between primary $(<4 \mathrm{~h})$ and secondary $(>4 \mathrm{~h})$ colonisation sub-microbiomes are representative of whether expression has increased or remained constant

Prevotellaceae showed the most transcriptional activity during primary colonisation, whilst the families Lachnospiraceae and Ruminococcaceae tended to be more transcriptionally active during secondary colonisation. The families Fibrobacteriaceae and Ruminococcaceae showed reasonably equal activity across primary and secondary colonisation events. These temporal shifts of families are in line with the 16S rRNA (based on cDNA) data already published for the same samples showing that RNAbased metataxonomy and metatranscriptome data findings were comparable [10]. Gene network analysis also showed that these dominant bacterial families were largely non-cooperative, as on a gene expression basis, they did not interact substantially with the two submicrobiomes, with the exception being Prevotellaceae. This is perhaps a consequence of the fact that their adaptation to this environment reduces the need to behave cooperatively and, therefore, could be characterised as 'selfish' [9]. Conversely, the less abundant bacterial families Burkholderiaceae, Enterobacteriaceae, Cyclobacteriaeceae and Flammeovirigaceae displayed highly cooperative behaviour in either the primary or secondary temporally distinct sub-microbiomes. This suggests that they may have an important role as keystone families for effective attachment and degradation of PRG.

Keystone bacteria are normally defined as species that if removed would have a negative effect on the structure and function of the community [53]. These four families are not high in abundance in the rumen [68] and were low in activity in this study. However, it seems they may play an integral role in their respective sub-microbiomes and possibly aid connectivity within the biofilms. Keystone taxa are often described as being cooperative and mutualistic, but in the context of plant colonisation in the rumen, the low levels of activity of these organisms suggest that they may be displaying commensalism by 'cheating' [69], perhaps capitalising on the activity of others in the sub-microbiomes. This strategy would ensure the community stays together, rewarding synergistic behaviours in the sub-microbiome, allowing harvesting of energy from the activities of other groups with relatively little input. These results also raise questions about the minimum rumen prokaryotic diversity required for effective energy harvesting, the importance of minor keystone species to this activity and whether the presence of the dominant genera alone would be more energetically efficient. Recent data showed that ruminants with less diverse rumen microbiomes, based on bacteria only, are more feed efficient [70], suggesting that degradation of fresh PRG may be equally as efficient in the presence of the dominant prokaryotic families Lachnospiraceae and Prevotellaceae alone and comparative to the situation when they are amongst a complex microbiome.

The underlying mechanisms driving competitive antagonism between primary and secondary associated sub-microbiomes likely include nutrient availability and production of chemicals by the microbes themselves 

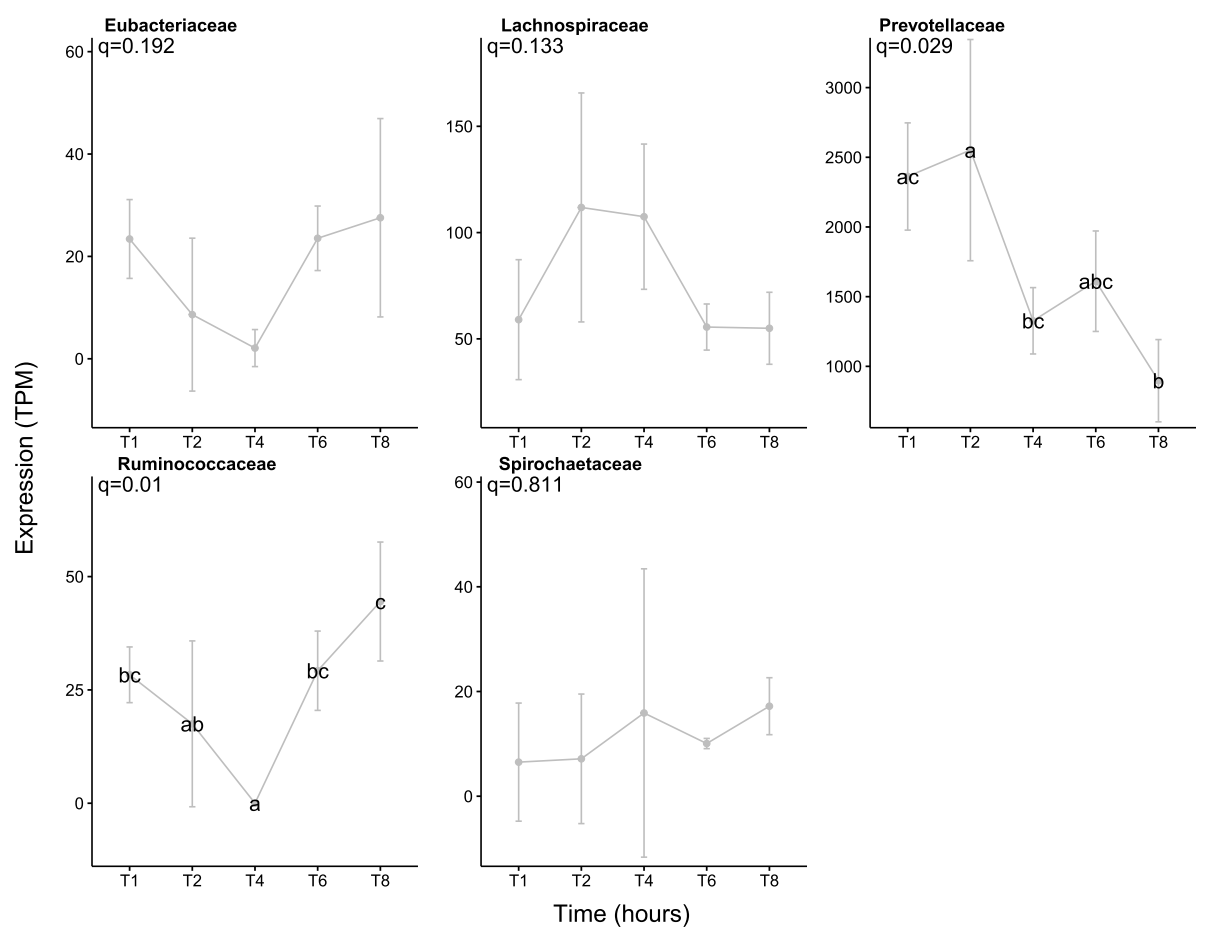

Fig. 8 Number of expressed LuxS genes (transcripts per million,TPM) for each prokaryotic taxonomic family colonising fresh perennial ryegrass incubated in the rumen over time. Incubation time is indicated on the axis, i.e. T1 indicates an incubation time of 1 h. The significance of incubation time is indicated on each plot, and where significance occurs then differences between timepoints are denoted by a different letter

[71]. During early rumen incubation $(<6 \mathrm{~h})$, fresh PRG is known to undergo self-mediated proteolysis and lipolysis, which will alter the form of the nutrients available to the rumen microbiome [72-74]. In parallel, it is known that PRG will be changing in terms of chemical content due to the rumen microbial breakdown of complex carbohydrates, lipids and proteins over time, thus providing a continually evolving niche for the microbes to inhabit $[6,10,75]$. In terms of plant nutrient degradation, we found that peptidase expression is high amongst the attached rumen prokaryotes, with limited DE seen between primary and secondary colonisation events. Most of the peptidase expression was by Prevotella, with species of this genera well known for their dominant proteolytic activity within the rumen microbiome [76, 77]. Carbohydrate breakdown was also a dominant activity of the fresh PRG attached bacteria, as denoted by the high expression of CAZymes/GHs. The preponderance of CAZyme activity within the rumen bacteria is well known and underpins their key function of energy harvesting by breaking down and fermenting complex carbohydrates to volatile fatty acids [78, 79]. Nonetheless, the temporal expression and contribution of each CAZyme family to fresh PRG has, to our knowledge, not been demonstrated before. We show that GH3, 5, 9, 10 and 48 dominate in terms of expression by the PRG-attached rumen bacteria, all of which are involved in the degradation of recalcitrant plant cell wall cellulose and hemicellulose, with all (all apart from GH48, which remains constant) slightly increasing over incubation time. Our data also highlight the niche specialisation and plasticity amongst the attached rumen bacteria as GH5 and 9 are expressed predominantly by Fibrobacter and/or Ruminococcus during primary colonisation but during secondary colonisation GH5 and 9 are predominantly expressed by Butyrivibrio and Pseudobutyrivibrio. This clearly demonstrates the redundancy, resilience and niche plasticity that occurs within the PRGattached rumen microbiome. We also demonstrate that $\mathrm{GH}$ family gene isoforms exist as has been shown previously [9] and that these within-family isoforms are differentially expressed over attachment time, further illustrating the redundancy that underpins the resilience of the rumen microbiome. Increases in methane metabolism were also apparent during secondary colonisation and this was linked to Butyrivibrio, Pseudobutyrivibrio and Methanobrevibacter activity. This is proposed to be due to increased hydrogen release from carbohydrate breakdown by the two bacteria genera being utilised by Methanobrevibacter to produce methane.

We also postulated that alongside known chemical changes in the fresh PRG [20], bacterial cell signalling and chemical warfare may also drive temporal niche specialisation. For example, in this study, we found that 
family Prevotellaceae and genus Prevotella, in particular, expressed AI-2 LuxS increasingly during primary colonisation and thereafter their expression during secondary colonisation declines. These data support the previous report that Prevotella were the most active in terms of expressing AI-2 LuxS genes in the rumen [17]. We also hypothesised that bacterial cell signalling may influence colonisation events and niche specialisation as it has previously been shown that furanosyl borate diester molecules encoded by the LuxS gene cause biofilm dispersal in many pure culture models [80]. Interestingly, no AHL genes were detected, which is consistent with other data, which suggests that AHL-based quorum sensing is low in the rumen [17]. Genes encoding the antimicrobial peptide (AMP) Lynronne 1 (likely produced by Prevotella ruminocola) were also upregulated during the primary colonisation phase of PRG and decreases in expression during the secondary phase [81]. In contrast, we showed that expression of non-ribosomally synthesised peptides and polyketide increased during secondary colonisation within fresh PRG attached bacteria [82]. The DE of these ribosomally and non-ribosomally synthesised AMPs and polyketides during the transition from primary to secondary colonisation may well contribute towards the antagonism between the bacteria characteristic of the two phases of PRG colonisation. This suggests that alongside mutualism, competition due to chemical warfare may be rife in the rumen and contribute to differences in niche occupancy over time. Interestingly, within these studies, expression of LuxS and ribosomally and non-ribosomally synthesised AMPs and polyketides was most pronounced within the genus Prevotella. Network analysis also showed that the family Prevotellaceae, of which we only identified the genus Prevotella, had more negative than positive interactions. However, it is likely that plant chemical changes, both self-induced and microbially mediated, alongside cell signalling and chemical warfare all collectively contribute towards the ecological plasticity seen within biofilms occurring at the plant interface.

In summary, our findings substantially expand our ecological understanding of microbial energy harvesting and niche development within the rumen. Furthermore, this study provides a major step change in our understanding of the microbial diversity and function required within a utopian microbiome for optimal host phenotype, whilst providing insight into the desired plant characteristics needed for maximum energy harvesting.

\section{Supplementary Information}

The online version contains supplementary material available at https://doi. org/10.1186/s40168-021-01087-w.

Additional file 1. Supplementary data and results.

\section{Acknowledgements}

Not Applicable

\section{Authors' contributions}

SAH, JEE, JAP and AKS conceived the project. PS and SAH completed the laboratory work. MA, DS and SC completed the library preparation and sequencing and CC, FR, WL completed downstream computational analysis with contribution from MW and LBO. SAH, CC, JEE and FR interpreted the results with contribution from $\mathrm{MW}$ and $\mathrm{LBO}$. All authors contributed to the preparation of the manuscript and approved its final version.

\section{Funding}

This work was supported by the Biotechnology and Biological Sciences Research Council Institute Strategic Programme Grant, Rumen Systems Biology (grant number BBS/E/W/10964), Core Strategic Programme in Resilient Crops (BBS/E/W/0012843D) and The Genome Analysis Centre (now the Earlham Institute) Capacity and Capability Challenge Programme.

\section{Availability of data and materials}

Reads of the sequence data have been deposited in the NCBI Short Read Archive under bioproject accession no. PRJNA419191. All scripts and intermediate data are deposited on the Open Science Framework at https:// osf.io/rx9h2/.

\section{Declarations}

\section{Ethics approval}

The experiment was conducted with the authority of licences under the United Kingdom Animal Scientific Procedures Act, 1986, and managed according to the protocols approved by the Aberystwyth University Animal Welfare and Ethics Review.

\section{Consent for publication \\ Not Applicable}

\section{Competing interests}

The authors declare no competing interests.

\section{Author details}

'Institute of Global Food Security, School of Biological Sciences, Queen's University Belfast, 19 Chlorine Gardens, Belfast BT9 5DL, Northern Ireland, UK. ${ }^{2}$ Institute of Biological, Environmental and Rural Sciences, Aberystwyth University, Aberystwyth SY23 3FG, UK. ${ }^{3}$ Laboratory of Microbiology, Wageningen University \& Research, 6708 Wageningen, WE, Netherlands. ${ }^{4}$ Current work address: Palital Feed Additives, Velddriel, Netherlands. ${ }^{5}$ Earlham Institute, Norwich NR4 7UH, UK. ${ }^{6}$ Quadram Institute, Norwich NR4 7UA, UK.

Received: 2 April 2021 Accepted: 2 May 2021

Published online: 21 June 2021

\section{References}

1. Banerjee S, Schlaeppi K, van der Heijden MGA. Keystone taxa as drivers of microbiome structure and functioning. Nat Rev Microbiol. 2018;16(9):56776. https://doi.org/10.1038/s41579-018-0024-1.

2. Sanders JG, Beichman AC, Roman J, Scott JJ, Emerson D, McCarthy JJ, et al. Baleen whales host a unique gut microbiome with similarities to both carnivores and herbivores. Nat Commun. 2015;6(1):8285. https://doi.org/10.1 038/ncomms9285.

3. Saterberg T, Jonsson T, Yearsley J, Berg S, Ebenman B. A potential role for rare species in ecosystem dynamics. Sci Rep. 2019;9(1):11107. https://doi. org/10.1038/s41598-019-47541-6.

4. Sonnenburg JL, Bäckhed F. Diet-microbiota interactions as moderators of human metabolism. Nature. 2016;535(7610):56-64. https://doi.org/10.1038/ nature18846.

5. Huws SA, Creevey CJ, Oyama LB, Mizrahi I, Denman SE, Popova M, et al. Addressing global ruminant agricultural challenges through understanding the rumen microbiome: past, present, and future. Front Microbiol. 2018;9: 2161. https://doi.org/10.3389/fmicb.2018.02161.

6. Edwards JE, Kingston-Smith AH, Jimenez HR, Huws SA, Skot KP, Griffith GW, et al. Dynamics of initial colonization of nonconserved perennial ryegrass by 
anaerobic fungi in the bovine rumen. FEMS Microbiol Ecol. 2008;66(3):53745. https://doi.org/10.1111/j.1574-6941.2008.00563.x.

7. Kingston-Smith AH, Edwards JE, Huws SA, Kim EJ, Abberton M. Plant-based strategies towards minimising 'livestock's long shadow'. Proc Nutr Soc. 2010;69(4):613-20. https://doi.org/10.1017/S0029665110001953.

8. Shabat SK, Sasson G, Doron-Faigenboim A, Durman T, Yaacoby S, Berg Miller ME, et al. Specific microbiome-dependent mechanisms underlie the energy harvest efficiency of ruminants. Isme J. 2016;10(12):2958-72. https:// doi.org/10.1038/ismej.2016.62.

9. Rubino F, Carberry C, Waters SM, Kenny D, Mc Cabe MS, Creevey CJ. Divergent functional isoforms drive niche specialisation for nutrient acquisition and use in rumen microbiome. Isme J. 2017;11(4):932-44. https://doi.org/10.1038/ismej.2016.172

10. Huws SA, Edwards JE, Creevey CJ, Rees Stevens P, Lin W, Girdwood SE, et al. Temporal dynamics of the metabolically active rumen bacteria colonizing fresh perennial ryegrass. FEMS Microbiol Ecol. 2016;92(1).

11. Huws SA, Mayorga OL, Theodorou MK, Onime LA, Kim EJ, Cookson AH, et al. Successional colonization of perennial ryegrass by rumen bacteria. Lett Appl Microbiol. 2013;56(3):186-96. https://doi.org/10.1111/lam.12033.

12. Leng RA. Interactions between microbial consortia in biofilms: a paradigm shift in rumen microbial ecology and enteric methane mitigation. Anim Prod Sci. 2014;54(5):519-43. https://doi.org/10.1071/AN13381.

13. McAllister TA, Bae HD, Jones GA, Cheng KJ. Microbial attachment and feed digestion in the rumen. J Anim Sci. 1994;72(11):3004-18. https://doi.org/10.2 527/1994.72113004x.

14. Elias S, Banin E. Multi-species biofilms: living with friendly neighbors. FEMS Microbiol Rev. 2012;36(5):990-1004. https://doi.org/10.1111/j.1574-6976.2012. 00325.x.

15. Nadell CD, Hartmann R, Drescher K. An emerging grip on the growth of grounded bacteria. ACS Nano. 2016;10(10):9109-10. https://doi.org/10.1 021/acsnano.6b06461.

16. Nadell CD, Xavier JB, Foster KR. The sociobiology of biofilms. FEMS Microbiol Rev. 2009;33(1):206-24. https://doi.org/10.1111/j.1574-6976.2008.00150.x.

17. Won MY, Oyama LB, Courtney SJ, Creevey CJ, Huws SA. Can rumen bacteria communicate to each other? Microbiome. 2020;8(1):23. https://doi.org/10.11 86/s40168-020-00796-y.

18. Edwards JE, Huws SA, Kim EJ, Lee MR, Kingston-Smith AH, Scollan ND. Advances in microbial ecosystem concepts and their consequences for ruminant agriculture. Animal. 2008;2(5):653-60.

19. Elliott $\mathrm{CL}$, Edwards JE, Wilkinson TJ, Allison GG, McCaffrey K, Scott MB, et al. Using omic approaches to compare temporal bacterial colonization of Lolium perenne, Lotus corniculatus, and Trifolium pratense in the rumen. Front Microbiol. 2018;9:2184. https://doi.org/10.3389/fmicb.2018.02184.

20. Mayorga OL, Kingston-Smith AH, Kim EJ, Allison GG, Wilkinson TJ, Hegarty MJ, et al. Temporal metagenomic and metabolomic characterization of fresh perennial ryegrass degradation by rumen bacteria. Front Microbiol. 2016;7:1854

21. Faust K, Raes J. Microbial interactions: from networks to models. Nat Rev Microbiol. 2012;10(8):538-50. https://doi.org/10.1038/nrmicro2832.

22. Pacheco AR, Segre D. A multidimensional perspective on microbial interactions. FEMS Microbiol Lett. 2019;366(11).

23. Chesson P. Mechanisms of maintenance of species diversity. Annu Rev Ecol Syst. 2000;31(1):343-66. https://doi.org/10.1146/annurev.ecolsys.31.1.343.

24. Leinweber A, Fredrik Inglis R, Kummerli R. Cheating fosters species coexistence in well-mixed bacterial communities. Isme J. 2017;11(5):1179-88. https://doi.org/10.1038/ismej.2016.195.

25. Ørskov ER, Hovell FDD, Mould F. The use of the nylon bag technique for the evaluation of feedstuffs. Trop An Prod. 1980;5(3):195-213.

26. Vanzant ES, Cochran RC, Titgemeyer EC. Standardization of in situ techniques for ruminant feedstuff evaluation. J Anim Sci. 1998;76(10):271729. https://doi.org/10.2527/1998.76102717x.

27. Ougham HJ, Davies TGE. Leaf development in Lolium temulentum: gradients of RNA complement and plastid and non-plastid transcripts. Physiol Plant. 1990;79(2):331-8. https://doi.org/10.1111/j.1399-3054.1990.tb06750.x.

28. Andrews S. FastQC: a quality control tool for high throughput sequence data; 2010.

29. Bolger AM, Lohse M, Usadel B. Trimmomatic: a flexible trimmer for Illumina sequence data. Bioinformatics. 2014;30(15):2114-20. https://doi.org/10.1093/ bioinformatics/btu170.

30. Rubino, F, Creevey CJ. MGkit: Metagenomic framework for the study of microbial communities. http://bitbucket.org/setsuna80/mgkit. 2014.
31. Cole JR, Wang Q, Fish JA, Chai B, McGarrell DM, Sun Y, et al. Ribosomal Database Project: data and tools for high throughput rRNA analysis. Nucleic Acids Res. 2014;42(Database issue):D633-42. https://doi.org/10.1093/nar/ gkt1244.

32. Langmead B, Salzberg SL. Fast gapped-read alignment with Bowtie 2. Nat Methods. 2012;9(4):357-9. https://doi.org/10.1038/nmeth.1923.

33. Byrne SL, Nagy I, Pfeifer M, Armstead I, Swain S, Studer B, et al. A syntenybased draft genome sequence of the forage grass Lolium perenne. Plant J. 2015;84(4):816-26. https://doi.org/10.1111/tpj.13037.

34. Seshadri R, Leahy SC, Attwood GT, Teh KH, Lambie SC, Cookson AL, et al. Cultivation and sequencing of rumen microbiome members from the Hungate1000 Collection. Nat Biotechnol. 2018;36(4):359-67. https://doi.org/1 $0.1038 /$ nbt.4110

35. Brown, $C T$, Howe A, Zhang Q, Pyrkosz AB, Brom TH. A reference-free algorithm for computational normalization of shotgun sequencing data. Unpublished preprint arxiv:1203.4802. arXiv pre-print server. 2012.

36. Zerbino DR, Birney E. Velvet: algorithms for de novo short read assembly using de Bruijn graphs. Genome Res. 2008;18(5):821-9. https://doi.org/10.11 01/gr.074492.107.

37. Haas BJ, Papanicolaou A, Yassour M, Grabherr M, Blood PD, Bowden J, et al. De novo transcript sequence reconstruction from RNA-seq using the Trinity platform for reference generation and analysis. Nat Protoc. 2013;8(8):1494512. https://doi.org/10.1038/nprot.2013.084.

38. Haas, BJ, Papanicolaou A. TransDecoder. Available from: https://github.com/ TransDecoder/TransDecoder/wiki. 2018.

39. Anders S, Pyl PT, Huber W. HTSeq--a Python framework to work with highthroughput sequencing data. Bioinformatics. 2015;31(2):166-9. https://doi. org/10.1093/bioinformatics/btu638.

40. Buchfink B, Xie C, Huson DH. Fast and sensitive protein alignment using DIAMOND. Nat Methods. 2015;12(1):59-60. https://doi.org/10.1038/nmeth.3176.

41. Halary S, Mclnerney JO, Lopez P, Bapteste E. EGN: a wizard for construction of gene and genome similarity networks. BMC Evol Biol. 2013;13(1):146. https://doi.org/10.1186/1471-2148-13-146.

42. Huerta-Cepas J, Szklarczyk D, Heller D, Hernandez-Plaza A, Forslund SK, Cook H, et al. eggNOG 5.0: a hierarchical, functionally and phylogenetically annotated orthology resource based on 5090 organisms and 2502 viruses. Nucleic Acids Res. 2019;47(D1):D309-D14. https://doi.org/10.1093/nar/gky1085.

43. Kanehisa M. Toward understanding the origin and evolution of cellular organisms. Protein Sci. 2019;28(11):1947-51. https://doi.org/10.1002/pro.3715.

44. Kanehisa M, Goto S. KEGG: Kyoto Encyclopedia of Genes and Genomes. Nucleic Acids Res. 2000;28(1):27-30. https://doi.org/10.1093/nar/28.1.27.

45. Kanehisa M, Sato Y, Furumichi M, Morishima K, Tanabe M. New approach for understanding genome variations in KEGG. Nucleic Acids Res. 2019;47(D1): D590-D5. https://doi.org/10.1093/nar/gky962.

46. Nicholls SM, Aubrey W, Edwards A, Grave KD, Huws S, Schietgat L, et al. Recovery of gene haplotypes from a metagenome. bioRxiv. 2019:223404.

47. Csardi G, Nepusz T. The igraph software package for complex network research. Int J Complex Syst. 2006;1695.

48. Gentleman, R, Carey V, Huber W, Hahne F. genefilter: methods for filtering genes from high-throughput experiments. R package version 1.58.1. 2017.

49. Warnes, GR, Bolker B, Bonebakker L, Gentleman R, Wolfgang LAH, Lumley T, et al. gplots: Various $R$ programming tools for plotting data. [http://cran.rproject.org/package=gplots]. 2009.

50. Love MI, Huber W, Anders S. Moderated estimation of fold change and dispersion for RNA-seq data with DESeq2. Genome Biol. 2014;15(12):550. https://doi.org/10.1186/s13059-014-0550-8.

51. Bastian M, Heymann S, Jacomy M, editors. Gephi: an open source software for exploring and manipulating networks: International AAAI Conference on Weblogs and Social Media; 2009.

52. Power ME, Tilman D, Estes JA, Menge BA, Bond WJ, Mills LS, et al. Challenges in the Quest for Keystones: Identifying keystone species is difficult-but essential to understanding how loss of species will affect ecosystems. BioScience. 1996;46(8):609-20. https://doi.org/10.2307/1312990.

53. Berry D, Widder S. Deciphering microbial interactions and detecting keystone species with co-occurrence networks. Front Microbiol. 2014;5:219.

54. Anders S, Huber W. Differential expression analysis for sequence count data. Genome Biol. 2010;11(10):R106. https://doi.org/10.1186/gb-201 0-11-10-r106.

55. Lombard V, Golaconda Ramulu H, Drula E, Coutinho PM, Henrissat B. The carbohydrate-active enzymes database (CAZy) in 2013. Nucleic Acids Res. 2014;42(Database issue):D490-5. https://doi.org/10.1093/nar/gkt1178. 
56. Yin $Y$, Mao X, Yang J, Chen X, Mao F, Xu Y. dbCAN: a web resource for automated carbohydrate-active enzyme annotation. Nucleic Acids Res. 2012; 40(Web Server issue):W445-51.

57. Rawlings ND, Barrett AJ, Thomas PD, Huang X, Bateman A, Finn RD. The MEROPS database of proteolytic enzymes, their substrates and inhibitors in 2017 and a comparison with peptidases in the PANTHER database. Nucleic Acids Res. 2018;46(D1):D624-D32. https://doi.org/10.1093/nar/gkx1134.

58. Sievers F, Higgins DG. Clustal Omega for making accurate alignments of many protein sequences. Protein Sci. 2018;27(1):135-45. https://doi.org/10.1 002/pro.3290.

59. Eddy SR. Accelerated profile HMM searches. PLoS Comput Biol. 2011;7(10): e1002195. https://doi.org/10.1371/journal.pcbi.1002195.

60. Edgar RC. MUSCLE: multiple sequence alignment with high accuracy and high throughput. Nucleic Acids Res. 2004;32(5):1792-7. https://doi.org/10.1 093/nar/gkh340.

61. Kozlov AM, Darriba D, Flouri T, Morel B, Stamatakis A. RAxML-NG: a fast, scalable and user-friendly tool for maximum likelihood phylogenetic inference. Bioinformatics. 2019;35(21):4453-5. https://doi.org/10.1093/ bioinformatics/btz305.

62. Letunic I, Bork P. Interactive Tree Of Life (iTOL) v4: recent updates and new developments. Nucleic Acids Res. 2019;47(W1):W256-W9. https://doi.org/1 0.1093/nar/gkz239.

63. Jacomy M, Venturini T, Heymann S, Bastian M. ForceAtlas2, a continuous graph layout algorithm for handy network visualization designed for the Gephi software. PLoS One. 2014;9(6):e98679. https://doi.org/10.1371/journal. pone.0098679.

64. Febria CM, Hosen JD, Crump BC, Palmer MA, Williams DD. Microbial responses to changes in flow status in temporary headwater streams: a cross-system comparison. Front Microbiol. 2015;6:522.

65. Lupatini M, AKA S, RJS J, Antoniolli ZI, de Siqueira Ferreira A, Kuramae EE, et al. Network topology reveals high connectance levels and few key microbial genera within soils. Front Env Sci. 2014;2(10).

66. Smith NW, Shorten PR, Altermann EH, Roy NC, McNabb WC. Hydrogen cross-feeders of the human gastrointestinal tract. Gut Microbes. 2019;10(3): 270-88. https://doi.org/10.1080/19490976.2018.1546522.

67. Williams RJ, Howe A, Hofmockel KS. Demonstrating microbial co-occurrence pattern analyses within and between ecosystems. Front Microbiol. 2014;5: 358.

68. Henderson G, Cox F, Ganesh S, Jonker A, Young W, Abecia L, et al. Rumen microbial community composition varies with diet and host, but a core microbiome is found across a wide geographical range. Sci Rep. 2015;5(1): 14567. https://doi.org/10.1038/srep14567.

69. Strassmann JE. Bacterial cheaters. Nature. 2000;404(6778):555-6. https://doi. org/10.1038/35007175

70. Li F, Guan LL. Metatranscriptomic profiling reveals linkages between the active rumen microbiome and feed efficiency in beef cattle. Appl Environ Microbiol. 2017:83(9).

71. Garcia-Bayona L, Comstock LE. Bacterial antagonism in host-associated microbial communities. Science. 2018;361(6408).

72. Beha EM, Theodorou MK, Thomas BJ, Kingston-Smith AH. Grass cells ingested by ruminants undergo autolysis which differs from senescence: implications for grass breeding targets and livestock production. Plant Cell Environ. 2002; 25(10):1299-312. https://doi.org/10.1046/j.1365-3040.2002.00908.x.

73. Kingston-Smith AH, Davies TE, Edwards JE, Theodorou MK. From plants to animals; the role of plant cell death in ruminant herbivores. J Exp Bot. 2008; 59(3):521-32. https://doi.org/10.1093/jxb/erm326.

74. Kingston-Smith AH, Merry RJ, Leemans DK, Thomas H, Theodorou MK. Evidence in support of a role for plant-mediated proteolysis in the rumens of grazing animals. Br J Nutr. 2005;93(1):73-9. https://doi.org/10.1079/BJN2 0041303.

75. Kingston-Smith AH, Davies TE, Rees Stevens P, Mur LA. Comparative metabolite fingerprinting of the rumen system during colonisation of three forage grass (Lolium perenne L.) varieties. PLoS One. 2013;8(11):e82801.

76. Attwood GT, Reilly K. Characterization of proteolytic activities of rumen bacterial isolates from forage-fed cattle. J Appl Bacteriol. 1996;81(5):545-52. https://doi.org/10.1111/j.1365-2672.1996.tb03545.x

77. Wallace RJ. Ruminal microbial metabolism of peptides and amino acids. J Nutr. 1996;126(4 Suppl):1326S-34S. https://doi.org/10.1093/jn/126. suppl_4.1326S.
78. Morais S, Mizrahi I. Islands in the stream: from individual to communal fiber degradation in the rumen ecosystem. FEMS Microbiol Rev. 2019;43(4):36279. https://doi.org/10.1093/femsre/fuz007.

79. Stewart RD, Auffret MD, Warr A, Walker AW, Roehe R, Watson M. Compendium of 4,941 rumen metagenome-assembled genomes for rumen microbiome biology and enzyme discovery. Nat Biotechnol. 2019;37(8):95361. https://doi.org/10.1038/s41587-019-0202-3.

80. Guilhen C, Forestier C, Balestrino D. Biofilm dispersal: multiple elaborate strategies for dissemination of bacteria with unique properties. Mol Microbiol. 2017;105(2):188-210. https://doi.org/10.1111/mmi.13698.

81. Oyama LB, Girdwood SE, Cookson AR, Fernandez-Fuentes N, Privé F, Vallin $H E$, et al. The rumen microbiome: an underexplored resource for novel antimicrobial discovery. npj Biofilms Microbiomes. 2017;3(1):33.

82. Moreira SM, de Oliveira Mendes TA, Santanta MF, Huws SA, Creevey CJ, Mantovani HC. Genomic and gene expression evidence of nonribosomal peptide and polyketide production among ruminal bacteria: a potential role in niche colonization? FEMS Microbiol Ecol. 2020;96(2).

\section{Publisher's Note}

Springer Nature remains neutral with regard to jurisdictional claims in published maps and institutional affiliations.
Ready to submit your research? Choose BMC and benefit from:

- fast, convenient online submission

- thorough peer review by experienced researchers in your field

- rapid publication on acceptance

- support for research data, including large and complex data types

- gold Open Access which fosters wider collaboration and increased citations

- maximum visibility for your research: over $100 \mathrm{M}$ website views per year

At BMC, research is always in progress.

Learn more biomedcentral.com/submissions 